\title{
Baseline clinical characteristics and disease burden in patients with paroxysmal nocturnal hemoglobinuria (PNH): updated analysis from the International PNH Registry
}

\author{
Hubert Schrezenmeier ${ }^{1,2}\left(\right.$ D $~ \cdot$ Alexander Röth $^{3} \cdot$ David J. Araten $^{4} \cdot$ Yuzuru Kanakura ${ }^{5} \cdot$ Loree Larratt $^{6}$. \\ Jamile M. Shammo ${ }^{7} \cdot$ Amanda Wilson $^{8,9} \cdot$ Gilda Shayan $^{8,10} \cdot$ Jaroslaw P. Maciejewski $^{11}$
}

Received: 28 May 2019 / Accepted: 21 April 2020 / Published online: 10 May 2020

(C) The Author(s) 2020

\begin{abstract}
The International Paroxysmal Nocturnal Hemoglobinuria (PNH) Registry (NCT01374360) was initiated to optimize patient management by collecting data regarding disease burden, progression, and clinical outcomes. Herein, we report updated baseline demographics, clinical characteristics, disease burden data, and observed trends regarding clone size in the largest cohort of Registry patients. Patients with available data as of July 2017 were stratified by glycosylphosphatidylinositol (GPI)-deficient granulocyte clone size $(<10 \%, \geq 10 \%-<50 \%$, and $\geq 50 \%)$. All patients were untreated with eculizumab at baseline, defined as date of eculizumab initiation or date of Registry enrollment (if never treated with eculizumab). Outcomes assessed in the current analysis included proportions of patients with high disease activity (HDA), history of major adverse vascular events (MAVEs; including thrombotic events [TEs]), bone marrow failure (BMF), red blood cell (RBC) transfusions, and PNH-related symptoms. A total of 4439 patients were included, of whom $2701(60.8 \%)$ had available GPI-deficient granulocyte clone size data. Among these, median clone size was $31.8 \%$ (1002 had $<10 \% ; 526 \mathrm{had} \geq 10 \%-<50 \% ; 1173 \mathrm{had} \geq 50 \%)$. There were high proportions of patients with HDA (51.6\%), history of MAVEs (18.8\%), BMF (62.6\%), RBC transfusion (61.3\%), and impaired renal function (42.8\%). All measures except RBC transfusion history significantly correlated with GPI-deficient granulocyte clone size. A large proportion of patients with GPI-deficient granulocyte clone size < 10\% had hemolysis (9.7\%), MAVEs (10.2\%), HDA (9.1\%), and/or PNH-related symptoms. Although larger GPI-deficient granulocyte clone sizes were associated with higher disease burden, a substantial proportion of patients with smaller clone sizes had history of MAVEs/TEs.
\end{abstract}

Statement of prior presentation: Portions of this work were presented at the American Society of Hematology 59th Annual Meeting, December 9-12, 2017, Atlanta, GA, USA.

Hubert Schrezenmeier

h.schrezenmeier@blutspende.de

Institute of Transfusion Medicine, University of Ulm, Ulm, Germany

2 Institute for Clinical Transfusion Medicine and Immunogenetics, German Red Cross Blood Transfusion Service Baden-Württemberg-Hessen and University Hospital Ulm, Helmholtzstraße 10, 89081 Ulm, Germany

3 Department of Hematology, West German Cancer Center, University Hospital Essen, University of Duisburg-Essen, Hufelandstr.55, D-45122 Essen, Germany

4 Department of Medicine, NYU Langone Medical Center, 550 First Avenue, 15-1539, New York, NY 10016, USA

5 Department of Hematology and Oncology, Osaka University Graduate School of Medicine, C9, 2-2, Yamada-oka, Suita, Osaka 565-0871, Japan
6 Department of Medicine, University of Alberta, Edmonton, Alberta, Canada

7 Division of Hematology/Oncology, Rush University Medical Center, 1653 W. Congress Parkway, Chicago, IL 60612, USA

8 Alexion Pharmaceuticals, Inc., 121 Seaport Boulevard, Boston, MA 02210, USA

9 Present address: Sanofi, 50 Binney Street, Boston, MA 02142, USA

10 Present address: Alnylam Pharmaceuticals, Inc., 675 West Kendall Street, Cambridge, MA 02142, USA

11 Department of Translational Hematology and Oncology Research, Taussig Cancer Institute, Cleveland Clinic, 10201 Carnegie Avenue, Cleveland, OH 44195, USA 
Keywords Paroxysmal nocturnal hemoglobinuria $\cdot$ Registries $\cdot$ Health-related quality of life $\cdot$ Eculizumab $\cdot$ Thrombosis $\cdot$ Bone marrow failure

\section{Introduction}

Paroxysmal nocturnal hemoglobinuria $(\mathrm{PNH})$ is a rare, acquired, potentially life-threatening hematologic disorder characterized by chronic, intravascular hemolysis caused by uncontrolled activation of the terminal complement pathway [1-3]. PNH is a clinically heterogeneous disease; in addition to the primary clinical manifestation of chronic intravascular hemolysis, it is associated with bone marrow failure (BMF; e.g., aplastic anemia, myelodysplastic syndrome) and thrombophilia [4-6]. Other disease manifestations include fatigue, abdominal pain, esophageal spasms, male erectile dysfunction, pulmonary hypertension, and renal impairment $[1,2$, 6-9].

Thrombotic events (TEs) account for up to $67 \%$ of deaths with a known cause in patients with PNH $[1,4,8,10,11]$. In a retrospective study, approximately $20 \%$ of patients with $\mathrm{PNH}$ treated only with supportive care died within 6 years of diagnosis [12].

PNH occurs as a consequence of a somatic mutation in the phosphatidylinositol glycan class A gene in bone marrow stem cells, followed by clonal expansion of the mutated cells. This genetic mutation causes impaired glycosylphosphatidylinositol (GPI) biosynthesis and deficient GPI-anchored complement regulatory proteins on the surface of mature blood cells $[4$, 13]. GPI-deficient granulocyte clone size, a measure of the relative proportion of GPI-deficient cells, varies widely among patients with PNH [5] and may influence clinical characteristics and disease burden [4-6]. Although patients with larger GPIdeficient granulocyte clone size generally experience more debilitating symptoms and consequences of chronic complementmediated intravascular hemolysis, limited data suggest that smaller GPI-deficient granulocyte clones may also confer significant disease burden [5, 6]. While larger GPI-deficient granulocyte clone size is associated with increased TE risk, all patients with PNH are at risk for a TE regardless of clone size [6, $11,14,15]$.

The International PNH Registry (NCT01374360) is the largest worldwide observational study of patients with PNH [6], designed to collect disease and treatment-related data that could be used to help optimize management of PNH $[16,17]$. Previous analyses of Registry data have demonstrated evidence of the clinical benefit of eculizumab (Soliris ${ }^{\circledR}$, Alexion Pharmaceuticals, Inc., Boston, MA, USA) for patients who have hemolysis and clinical symptoms indicative of high disease activity (HDA) [16]. Baseline demographic and clinical characteristics of the first 1610 patients enrolled in the Registry have also been previously reported [6].
The objectives of the present analysis are to provide an update on the baseline demographics, clinical characteristics, disease-associated morbidities, PNH symptoms, and healthrelated QoL in patients, based on a substantially larger cohort of Registry patients, and to assess potential trends in PNH disease burden related to GPI-deficient clone size [6].

\section{Methods}

\section{Study design and patients}

Details regarding the design and patient population included in the International PNH Registry have been previously reported [6]. The Registry is an ongoing, prospective, multicenter, global, observational study that includes patients of any age with a clinical diagnosis of PNH and/or a detectable PNH clone (defined as a population of GPI-deficient granulocytes and/or erythrocytes) of $\geq 0.01 \%$ [6]. The institutional review boards (or equivalent) of participating centers approved the Registry, and written informed consent was provided by all patients before study entry [6]. The Registry is sponsored by Alexion Pharmaceuticals, Inc., and is overseen by an Executive Committee of independent international clinical experts in PNH [6].

The current analysis includes patients enrolled in the Registry who had data available as of July 17, 2017. All patients were untreated with eculizumab at baseline, defined as the date of eculizumab initiation for patients who had ever been treated with eculizumab and the date of Registry enrollment for patients who had never received eculizumab treatment.

\section{Outcomes}

Clinical outcomes of interest assessed at baseline included proportions of patients with HDA, history of major adverse vascular events (MAVEs; including TEs) and BMF (including aplastic anemia), red blood cell (RBC) transfusions, concomitant medication use, and presence of physician-reported PNH-related symptoms. TEs include thrombophlebitis/deep vein thrombosis, renal vein thrombosis, renal arterial thrombosis, mesenteric/visceral vein thrombosis, mesenteric/ visceral arterial thrombosis, hepatic/portal vein thrombosis, dermal thrombosis, acute peripheral vascular disease occlusion, cerebral arterial occlusion/cerebrovascular accident, cerebral venous occlusion, and pulmonary embolus. MAVEs include TEs as defined above, amputation (nontraumatic, 
nondiabetic), myocardial infarction, transient ischemic attack, unstable angina, gangrene (nontraumatic, nondiabetic), and other MAVE. Characteristics such as aplastic anemia, hypoplastic anemia, myelodysplastic syndrome, and other bone marrow pathologies were used as a basis to identify history of or concurrent bone marrow failure in Registry patients. Laboratory values were assessed at baseline, defined as the most recent value within 6 months prior to and including the baseline date. HDA at baseline was defined as evidence of hemolysis (elevated lactate dehydrogenase $[\mathrm{LDH}] \geq 1.5$ times upper limit of normal [ULN]) within 6 months prior to baseline and a history of at least 1 of the following signs or symptoms prior to baseline: fatigue, hemoglobinuria, abdominal pain, dyspnea, anemia (hemoglobin $<100 \mathrm{~g} / \mathrm{L}$ ), MAVEs (including TEs), dysphagia, or erectile dysfunction.

Patient QoL was assessed using the European Organisation for Research and Treatment of Cancer (EORTC) Global Health/QoL subscale, which is rated on a scale ranging from 0 to 100 , with higher scores indicating better functioning [18]. Patient-reported fatigue was assessed using the Functional Assessment of Chronic Illness Therapy (FACIT)-Fatigue questionnaire, which is scored on a scale ranging from 0 to 52 , with higher scores indicating less fatigue $[19,20]$. For QoL measures, baseline was defined as the most recent value within 6 months prior to and including the baseline date.

\section{Data analysis}

Categorical variables were described using frequencies and percentages, and continuous variables were described using mean, standard deviation (SD), median, and interquartile range. For each outcome of interest, analyses were conducted only on the patients who had available data.

Patients whose percent GPI-deficient granulocyte clone size values were available prior to baseline were stratified by clone size category $(<10 \%, \geq 10 \%$ to $<50 \%$, and $\geq 50 \%)$. Potential trends related to clone size were assessed using the Cochran-Armitage trend test of proportion of patients with specified event history by percent of GPI-deficient granulocyte group.

\section{Results}

As of July 17, 2017, a total of 4948 patients were enrolled in the Registry. Of these, 4439 patients had nonmissing data on demographics, enrollment date, and eculizumab status and were included in the study population. Patient demographics are summarized in Table 1. Among the 2701 patients (60.8\%) with clone size reported prior to baseline, median (quartile [Q]1, Q3) percent GPI-deficient granulocyte clone size was $31.8 \%$ ( $2.4 \%, 85.0 \%)$. Of those, 1002 patients $(37.1 \%)$ had GPI-deficient granulocyte clone size $<10 \%, 526(19.5 \%)$ had clone size $\geq 10 \%$ to $<50 \%$, and $1173(43.4 \%)$ had clone size $\geq 50 \%$. More than half of the patients $(51.6 \%)$ had HDA at baseline, and the proportion of patients with HDA at baseline correlated significantly with GPI-deficient granulocyte clone size category; higher proportions of patients with HDA were observed in subgroups with higher clone size $(P<0.0001)$. However, high proportions of patients with small $(<10 \%)$ or mid-size ( $\geq 10 \%$ to $<50 \%$ ) GPI-deficient granulocyte clones had HDA ( $9 \%$ and $42 \%$, respectively) (Fig. 1).

Overall, 779 of 4134 patients (18.8\%) with available data at baseline had a history of MAVEs, including 544 (13.3\% of the study population) who specifically experienced TEs. The proportions of patients with a history of MAVEs or TEs at baseline correlated significantly with clone size (Fig. 2a; $P<0.0001$ for both MAVE and TE). While the proportions of patients with a history of MAVEs and TEs were substantially higher in the cohort with clone size $\geq 50 \%$ versus the cohort with clone size $\geq 10 \%$ to $<50 \%$, they were only modestly higher in the cohort with clone size $\geq 10 \%$ to $<50 \%$ versus the $<10 \%$ cohort.

Almost $63 \%$ of patients had a history of BMF and about $53 \%$ (2206/4201) had a history of aplastic or hypoplastic anemia at baseline. The proportions of patients with BMF in each of the cohorts showed an inverse correlation with clone size (Fig. 2b).

Overall, patients had a high burden of disease at baseline, as reflected in the proportions of patients with hemolysis $(55.8 \%)$, defined by an LDH ratio $\geq 1.5$ times ULN at baseline, and impaired renal function $(42.8 \%$ with estimated glomerular filtration rate $[\mathrm{eGFR}]<90 \mathrm{~mL} / \mathrm{min} / 1.73 \mathrm{~m}^{2}$ and $15.0 \%$ with eGFR $<60 \mathrm{~mL} / \mathrm{min} / 1.73 \mathrm{~m}^{2}$ ). Median values for all laboratory parameters assessed correlated significantly with GPI-deficient granulocyte clone size category (Table 2). The proportion of patients with impaired renal function (eGFR $<90 \mathrm{~mL} / \mathrm{min} / 1.73 \mathrm{~m}^{2}$ ) was highest in the cohort with clone size $<10 \%$ (51.4\%), compared with those with GPIdeficient granulocyte clone sizes of $\geq 10 \%$ to $<50 \%$ (40.5\%) and $\geq 50 \%$ (35.0\%). Conversely, median laboratory values at baseline for LDH ratio, absolute reticulocyte count, and platelet count showed a significant positive correlation with increasing GPI-deficient granulocyte clone size category (Table 2). There was a significant trend in median hemoglobin levels across GPI-deficient granulocyte clone size cohorts, with the lowest median hemoglobin level observed in the cohort with clone size $\geq 50 \%$.

In general, $61.3 \%(2219 / 3620)$ of patients had a history of RBC transfusions, $20.2 \%(849 / 4206)$ had a history of anticoagulation therapy, and 38.8\% (1642/4232) had a history of immunosuppressive therapy at baseline (Table 2). History of anticoagulant use and history of immunosuppressive therapy were significantly correlated with clone size $(P<0.0001)$, with the proportions of patients with history of anticoagulant use at baseline showing positive correlation with increasing 
Table 1 Patient demographic characteristics

\begin{tabular}{lll}
\hline Characteristic & $\begin{array}{l}\text { Overall study population } \\
(n=4439)\end{array}$ & $\begin{array}{l}\text { Patients with GPI-deficient granulocytes data } \\
\text { available at baseline } \\
(n=2701)\end{array}$ \\
\hline Sex, $n(\%)$ & $n=4439$ & $n=2701$ \\
Female & $2353(53.0)$ & $1407(52.1)$ \\
Male & $2086(47.0)$ & $1294(47.9)$ \\
Region, $n(\%)$ & $n=4439$ & - \\
Europe & $3012(67.9)$ & - \\
North America & $640(14.4)$ & - \\
Rest of world & $787(17.7)$ & - \\
Race, $n(\%)$ & $n=4420$ & $n=2692$ \\
White & $3464(78.4)$ & $2238(83.1)$ \\
Asian & $722(16.3)$ & $320(11.9)$ \\
Black & $132(3.0)$ & $83(3.1)$ \\
Other & $102(2.3)$ & $51(1.9)$ \\
Age at baseline, years & $n=4439$ & $n=2701$ \\
Mean (SD) & $45.1(18.1)$ & $44.4(18.6)$ \\
Median (Q1, Q3) & $43.7(30.2,59.6)$ & $42.7(28.6,59.8)$ \\
Age at disease onset, years & $n=4336$ & $n=2667$ \\
Mean (SD) & $39.3(18.6)$ & $40.0(19.0)$ \\
Median (Q1, Q3) & $35.5(23.9,53.4)$ & $36.5(23.8,55.2)$ \\
\hline
\end{tabular}

$Q$ quartile; $S D$ standard deviation

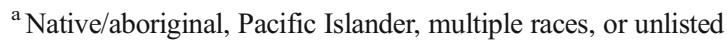

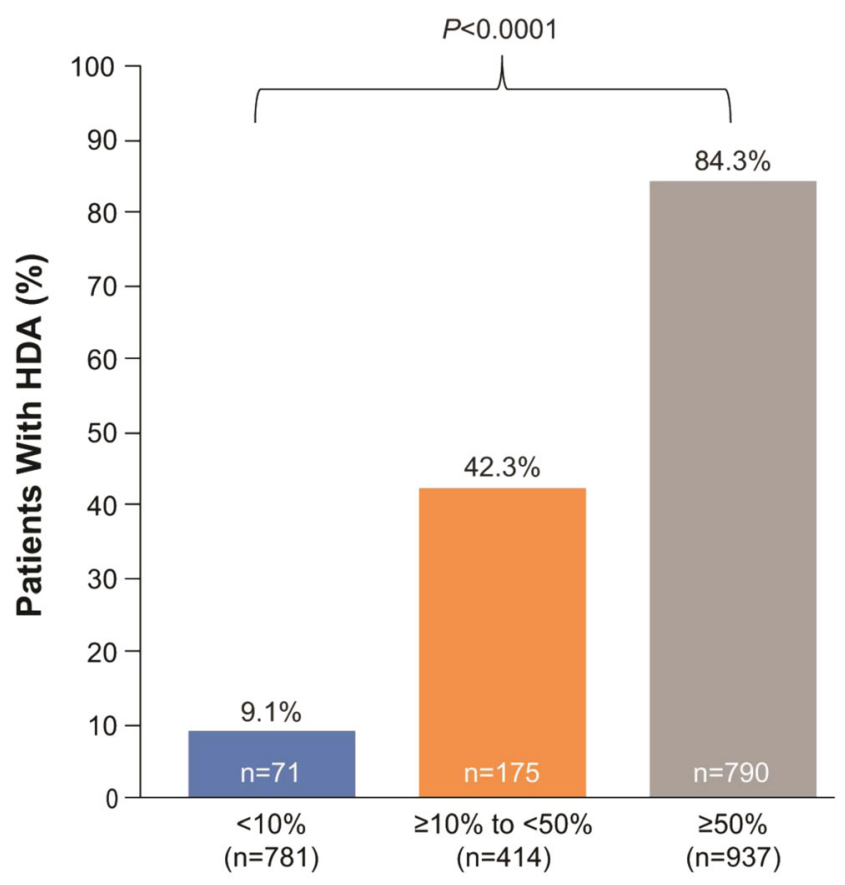

\% GPI-Deficient Granulocytes at Baseline ${ }^{a}$

Fig. 1 Proportion of patients with HDA stratified by percentage of GPIdeficient granulocytes at baseline ${ }^{\text {a }}$ Patients with available data. GPI glycosylphosphatidylinositol; HDA high disease activity clone size and proportions of patients with a history of immunosuppressive therapy showing inverse correlation with GPIdeficient granulocyte clone size category (Table 2). There was no statistically significant trend in the proportion of patients with a history of RBC transfusions across the three cohorts.

Substantial proportions of patients had a history of physician-reported PNH-related symptoms at baseline, including fatigue $(80.9 \%$ [2684/3318]), dyspnea $(45.3 \%$ [1501/3315]), hemoglobinuria (45.0\% [1492/3313]), abdominal pain (35.2\% [1167/3314]), dysphagia (16.5\% [547/ 3311]), and erectile dysfunction (24.2\% [344/1422 males]. Proportions of patients with PNH-related symptoms stratified by GPI-deficient granulocyte clone size at baseline are shown in Fig. 3. Although the proportion of patients with a history of each commonly reported PNH-related symptom was greatest in the subgroup with the largest GPI-deficient granulocyte clone size, a considerable proportion of patients with smaller clone sizes also experienced these symptoms.

In the overall population, patient-reported QoL assessments based on EORTC Global Health/QoL ( $n=1888$ patients with available data) and FACIT-Fatigue $(n=1894$ patients with available data) scores indicated impaired QoL and fatigue, with median (Q1, Q3) scores of $58.3(41.7,75.0)$ and 34.0 (27.0, 40.0), respectively. Patient-reported QoL assessments did not appear to correlate with GPI-deficient 
Fig. 2 Proportion of patients with a history of (a) MAVEs, TEs (a subset of all MAVEs), or (b) BMF stratified by percentage of GPIdeficient granulocytes at baseline BMF, $n=964$. ' MAVEs, $n=494$; ${ }^{\mathrm{c}}$ MAVEs, $n=1114$; TEs, $n=$ 1106; BMF, $n=1125$; ${ }^{\mathrm{d}}$ Patients with available data. $B M F$ bone marrow failure; $G P I$ glycosylphosphatidylinositol; MAVEs major adverse vascular events; TEs thrombotic events aMAVEs, $n=915$; TEs, $n=899$; TEs, $n=495 ; \mathrm{BMF}=509$.

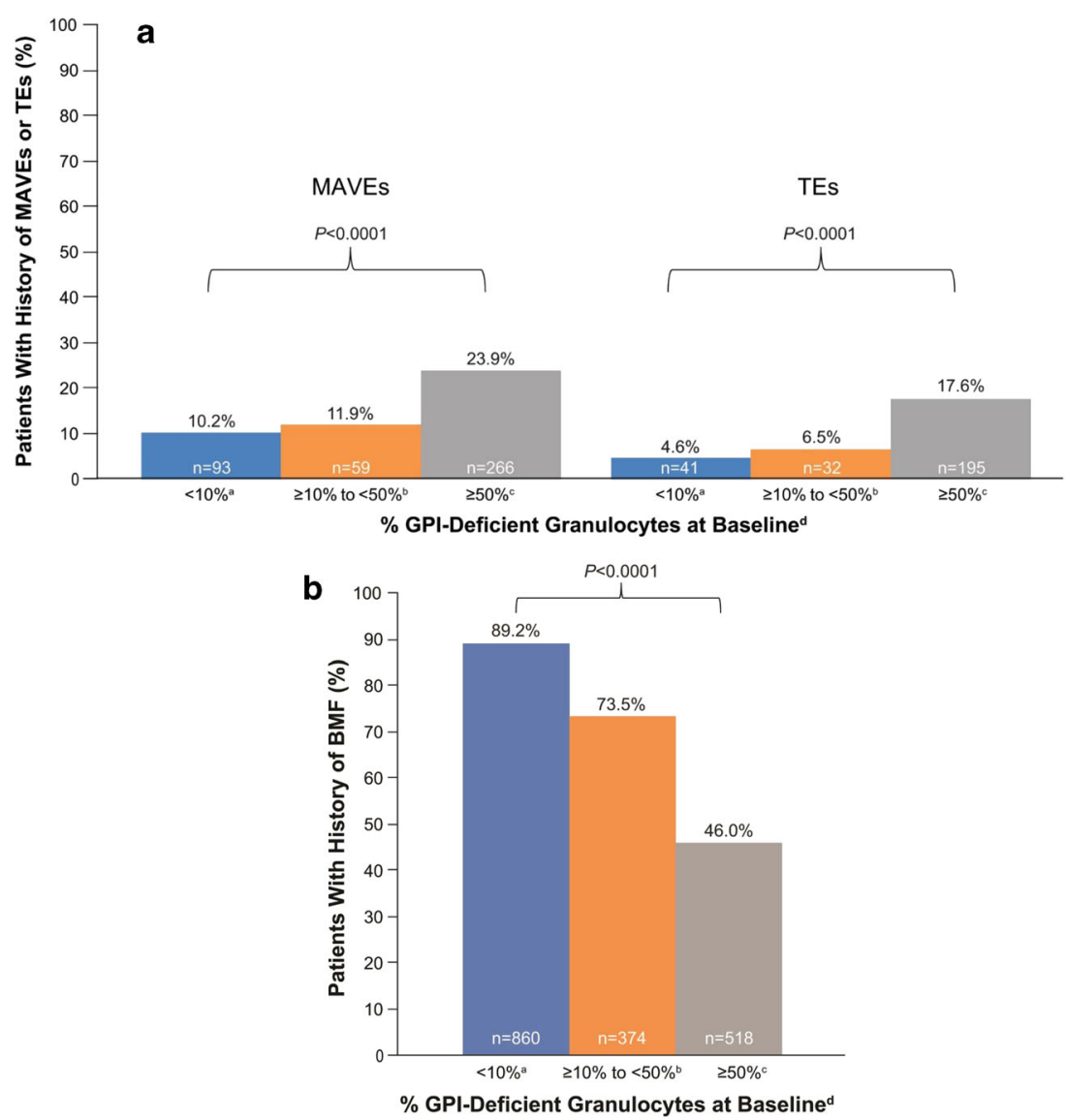

granulocyte clone size. Median (Q1, Q3) FACIT-Fatigue scores $(n=1317$ patients with available data) were 35.0 $(28.0,40.0)$ in patients with clone size $<10 \%, 37.0(28.0$, 41.0 ) in those with clone size $\geq 10 \%$ to $<50 \%$, and 32.0 $(23.0,39.0)$ in those with clone size $\geq 50 \%$; median (Q1, Q3) EORTC Global Health/QoL scores $(n=1310$ patients with available data) were $58.3(41.7,75.0), 58.3$ (50.0, $83.3)$, and $50.0(33.3,66.7)$, respectively.

\section{Discussion}

The current study provides updated data from the International PNH Registry describing baseline characteristics and disease burden in a much larger cohort of patients $(n=4439)$ than previously reported $(n=1610)$ [6]. While the burden and/or natural history of PNH has been documented in smaller cohorts $[1,3,6,11,15,17,21-24]$, the current analysis characterized the largest cohort of patients with PNH studied to date and examined trends in the relationship between disease burden and GPI-deficient granulocyte clone size.

The demographics and clinical history of the current study population were generally similar to the subset reported by Schrezenmeier et al. [6], although median age at PNH onset was slightly older and median duration of disease was slightly shorter in the current study. Median GPI-deficient granulocyte clone size was $31.8 \%$ in the present study compared with $68.1 \%$ in the earlier analysis of the Registry. In addition, our cohort had a higher proportion of patients with GPI-deficient granulocyte clone size $<10 \%$ compared with the previous study (37.1\% versus $17.4 \%$, respectively) and a smaller proportion of patients with clone size $\geq 50 \%$ ( $43.4 \%$ versus $51.7 \%$, respectively) [6]. It is likely that more patients with small GPI-deficient granulocyte clone sizes have been enrolled in the Registry in recent years due to improvements in disease awareness, enhanced emphasis on screening (e.g., screening for GPI-deficient cells is now routine in patients with aplastic anemia) and early detection, and the increased availability and utilization of high-sensitivity flow cytometry for detection of PNH clones $[4,6,25,26]$.

Our findings confirmed that PNH is associated with a high burden of disease, as demonstrated by the proportion of patients who had HDA (51.6\%), history of MAVEs (18.8\%), TEs (13.3\%), RBC transfusions (61.3\%), and impaired renal function (eGFR $<90 \mathrm{~mL} / \mathrm{min} / 1.73 \mathrm{~m}^{2}, 42.8 \%$ ) at baseline. Although higher GPI-deficient granulocyte clone size correlated with a higher proportion of patients with HDA and history of MAVEs, a considerable proportion of patients in the cohorts with GPI-deficient granulocyte clone size $\geq 10 \%$ to $<$ $50 \%$ and $<10 \%$ also had HDA and/or history of MAVEs, 
Table 2 Laboratory values and concomitant medication use at baseline

\begin{tabular}{|c|c|c|c|c|c|}
\hline \multirow[t]{2}{*}{ Parameter } & \multirow{2}{*}{$\begin{array}{l}\text { All Patients } \\
n=4439\end{array}$} & \multicolumn{4}{|c|}{ Patients by $\%$ GPI-deficient granulocytes at baseline ${ }^{a}$} \\
\hline & & $\begin{array}{l}<10 \% \\
n=1002\end{array}$ & $\begin{array}{l}\geq 10 \% \text { to }<50 \% \\
n=526\end{array}$ & $\begin{array}{l}\geq 50 \% \\
n=1173\end{array}$ & $P$ value \\
\hline $\mathrm{LDH}$ ratio (LDH/ULN) & $n=3085$ & $n=781$ & $n=414$ & $n=937$ & \\
\hline Median (Q1, Q3) & $1.9(1.0,5.1)$ & $0.9(0.7,1.1)$ & $1.4(1.0,2.3)$ & $4.6(2.6,7.3)$ & $<0.0001$ \\
\hline $\mathrm{LDH} \geq 1.5 \times \mathrm{ULN}, n(\%)$ & $1720(55.8)$ & $76(9.7)$ & $200(48.3)$ & $834(89.0)$ & $<0.0001$ \\
\hline $\begin{array}{l}\text { Hemoglobin }(\mathrm{g} / \mathrm{L}) \\
\text { Median }(\mathrm{Q} 1, \mathrm{Q} 3)\end{array}$ & $\begin{array}{l}n=3581 \\
98.0(82.2,117.0)\end{array}$ & $\begin{array}{l}n=914 \\
99.4(80.0,119.0)\end{array}$ & $\begin{array}{l}n=458 \\
101.0(87.0,124.0)\end{array}$ & $\begin{array}{l}n=1020 \\
95.0(81.0,108.0)\end{array}$ & $<0.0001$ \\
\hline $\begin{array}{l}\text { Absolute reticulocyte count }\left(\times 10^{9} / \mathrm{L}\right) \\
\text { Median }(\mathrm{Q} 1, \mathrm{Q} 3)\end{array}$ & $\begin{array}{l}n=1954 \\
87.0(55.0,140.0)\end{array}$ & $\begin{array}{l}n=444 \\
56.0(38.0,75.0)\end{array}$ & $\begin{array}{l}n=292 \\
75.0(51.5,105.5)\end{array}$ & $\begin{array}{l}n=664 \\
124.0(82.0,183.0)\end{array}$ & $<0.0001$ \\
\hline $\begin{array}{l}\text { Platelet count }\left(\times 10^{9} / \mathrm{L}\right) \\
\text { Median }(\mathrm{Q} 1, \mathrm{Q} 3)\end{array}$ & $\begin{array}{l}n=3563 \\
110.0(50.0,175.0)\end{array}$ & $\begin{array}{l}n=884 \\
59.5(23.0,135.0)\end{array}$ & $\begin{array}{l}n=449 \\
81.0(37.0,146.0)\end{array}$ & $\begin{array}{l}n=1026 \\
129.0(70.0,191.0)\end{array}$ & $<0.0001$ \\
\hline $\begin{array}{l}\mathrm{eGFR}\left(\mathrm{mL} / \mathrm{min} / 1.73 \mathrm{~m}^{2}\right), n(\%) \\
<30\end{array}$ & $\begin{array}{l}n=3257 \\
79(2.4)\end{array}$ & $\begin{array}{l}n=849 \\
12(1.4)\end{array}$ & $\begin{array}{l}n=430 \\
5(1.2)\end{array}$ & $\begin{array}{l}n=934 \\
15(1.6)\end{array}$ & $<0.0001$ \\
\hline $30-<60$ & $410(12.6)$ & $122(14.4)$ & $47(10.9)$ & $97(10.4)$ & \\
\hline $60-<90$ & $904(27.8)$ & $302(35.6)$ & $122(28.4)$ & $215(23.0)$ & \\
\hline$\geq 90$ & $1864(57.2)$ & $413(48.6)$ & $256(59.5)$ & $607(65.0)$ & \\
\hline History of RBC transfusions, $n(\%)$ & $n=3620$ & $n=979$ & $n=476$ & $n=966$ & 0.1204 \\
\hline & $2219(61.3)$ & $589(60.2)$ & $259(54.4)$ & $615(63.7)$ & \\
\hline History of anticoagulation therapy ${ }^{\mathrm{b}}, n(\%)$ & $\begin{array}{l}n=4206 \\
849(20.2)\end{array}$ & $\begin{array}{l}n=952 \\
49(5.1)\end{array}$ & $\begin{array}{l}n=499 \\
58(11.6)\end{array}$ & $\begin{array}{l}n=1127 \\
375(33.3)\end{array}$ & $<0.0001$ \\
\hline History of immunosuppressive therapy ${ }^{\mathrm{c}}, n(\%)$ & $\begin{array}{l}n=4232 \\
1642(38.8)\end{array}$ & $\begin{array}{l}n=971 \\
573(59.0)\end{array}$ & $\begin{array}{l}n=500 \\
212(42.4)\end{array}$ & $\begin{array}{l}n=1137 \\
302(26.6)\end{array}$ & $<0.0001$ \\
\hline
\end{tabular}

eGFR estimated glomerular filtration rate; GPI glycosylphosphatidylinositol; $L D H$ lactate dehydrogenase; $Q$ quartile; $R B C$ red blood cell; $U L N$ upper limit of normal

${ }^{a}$ Patients with available data. ${ }^{\mathrm{b}}$ Aspirin, warfarin derivatives, heparin derivatives, or other anticoagulation. ${ }^{\mathrm{c}}$ Corticosteroids, cyclosporine, or antithymocyte globulin

suggesting the need for further emphasis on disease symptoms in clinical decision-making and potential consideration of treatment in these populations.

The current analysis also examined the association of HDA with GPI-deficient granulocyte clone size, which was not previously assessed by Schrezenmeier et al. [6], and may be an important consideration in patient management. While more than half of the patients in this study had HDA [16], which was significantly and positively related to GPI-deficient granulocyte clone size, a considerable number of patients with small GPI-deficient granulocyte clone size also had HDA (9\%). This finding is clinically relevant considering that previous research has demonstrated that presence of HDA is related to poorer outcomes and higher risk of TE [15, 16, 27].

The proportion of patients with a history of BMF was higher in this study compared with the previous study (62.6\% versus $48.1 \%$, respectively), potentially owing to the higher proportion of patients with GPI-deficient granulocyte clone size $<10 \%$ in the current study cohort as discussed above [6]. This observation is consistent with previous research showing that a majority of patients with concurrent
PNH and BMF have GPI-deficient granulocyte clone sizes smaller than $10 \%$, with less than $10 \%$ of patients with $\mathrm{PNH} /$ BMF having a clone size larger than 50\% [28]. Of note, immunosuppressive therapy was reported in $59.0 \%$ of patients with GPI-deficient granulocyte clone size $<10 \%$, and patients in this group had lower reticulocyte counts versus those with larger clone sizes, which is not unexpected given the high proportion of patients with a history of BMF in this group $[13,22,29]$. Interestingly, the cohort with the smallest GPIdeficient granulocyte clone sizes also had a higher proportion of patients with renal failure at baseline compared with the larger clone-size groups. This is probably due to the fact that the patients in the smallest GPI-deficient granulocyte clonesize group had the highest prevalence of concomitant BMF (89\%) and immunosuppressive therapy use (59\%) at baseline.

In the current analysis, $61.3 \%$ of patients had a history of RBC transfusions, compared with approximately $36 \%$ of patients in the previous Registry analysis [6]. This finding may be related to the higher proportion of patients with a history of BMF in the current study population, who often require supportive treatment with RBC transfusions [5]. However, we 


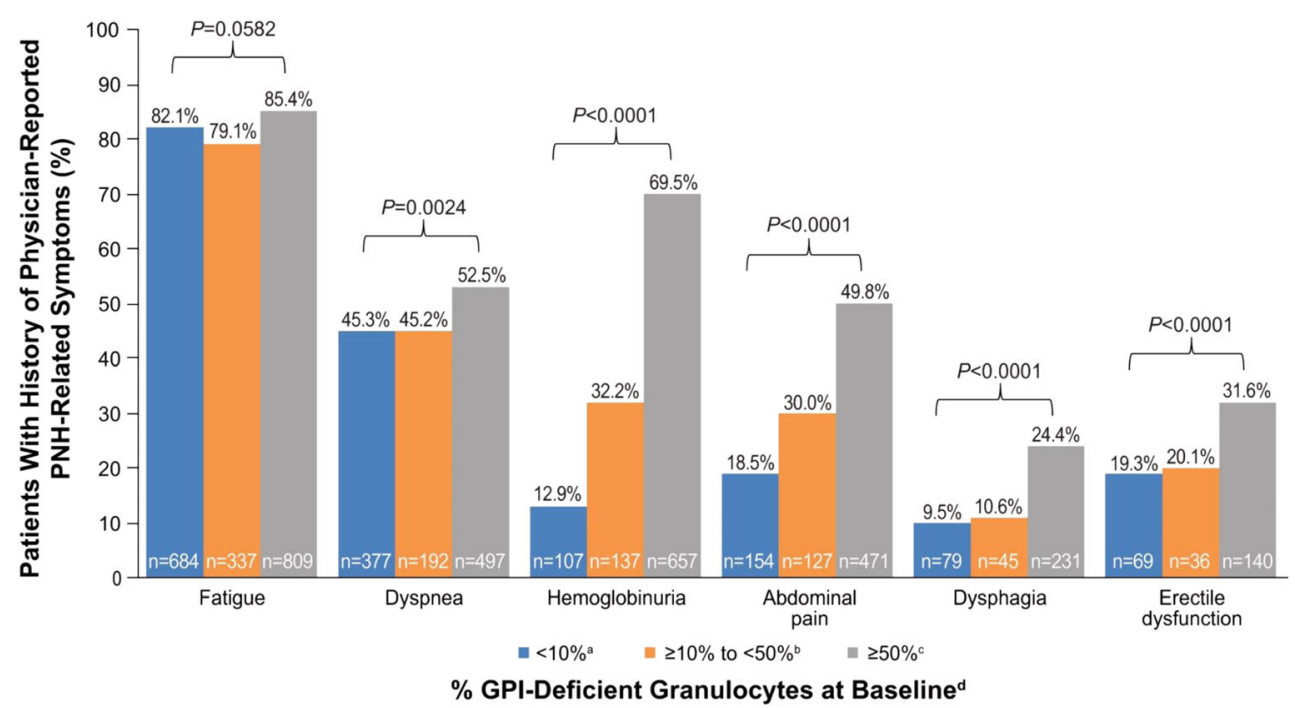

Fig. 3 History of physician-reported PNH-related symptoms stratified by percentage of GPI-deficient granulocytes at baseline a Fatigue, $n=833$; dyspnea, $n=832$; hemoglobinuria, $n=829$; abdominal pain, $n=832$; dysphagia, $n=830$; erectile dysfunction, $n=357$. ${ }^{\mathrm{b}}$ Fatigue, $n=426$; dyspnea, $n=425$; hemoglobinuria, $n=425$; abdominal pain, $n=424$;

found no significant association between history of RBC transfusions and clone size, and more than half of the patients in all clone size groups had experienced RBC transfusions at baseline. It can be hypothesized that patients with larger GPIdeficient granulocyte clone size may receive RBC transfusions to treat anemia related to the hemolysis of $\mathrm{PNH}$, whereas patients with a smaller clone size, who are more likely to have $\mathrm{BMF}$, may receive transfusions to treat anemia associated with BMF [5].

A large proportion of patients had hemolysis (55.8\%) and impaired renal function (42.8\%) at baseline, and most laboratory values indicative of greater disease burden were positively correlated with GPI-deficient granulocyte clone size. Median LDH ratio and the proportion of patients with LDH $\geq 1.5$ times ULN were significantly correlated to GPIdeficient granulocyte clone size, consistent with findings from Schrezenmeier et al. [6]. Interestingly, in our cohort, the proportions of patients with eGFR 30 to $<90$ decreased with increasing GPI-deficient granulocyte clone size, whereas the proportions of patients with eGFR $<30$ remained similar across different clone sizes. Although prior literature has demonstrated that renal dysfunction, including chronic kidney disease (CKD), is common among patients with $\mathrm{PNH}$, additional analyses are needed to better understand the relationship between PNH and CKD and the impact of concurrent or previous nephrotoxic therapy (i.e., cyclosporine and treatment of BMF), and to identify potential trends related to GPI-deficient granulocyte clone size [30, 31].

A substantial proportion of patients in all GPI-deficient granulocyte clone size categories experienced physician- dysphagia, $n=426$; erectile dysfunction, $n=179$. ' Fatigue, $n=947$; dyspnea, $n=947$; hemoglobinuria, $n=946$; abdominal pain, $n=945$; dysphagia, $n=946$; erectile dysfunction, $n=443$; ${ }^{\mathrm{d}}$ Patients with available data. GPI glycosylphosphatidylinositol; $P N H$ paroxysmal nocturnal hemoglobinuria

reported PNH-related symptoms such as fatigue, dyspnea, hemoglobinuria, abdominal pain, dysphagia, and erectile dysfunction. With the exception of fatigue, the proportions of patients with each symptom were significantly correlated with increasing GPI-deficient granulocyte clone size category, although a large proportion of patients with clone size $<50 \%$ experienced each symptom. This data pattern is similar to that observed by Schrezenmeier et al. [6], with the exception of dyspnea and abdominal pain, which were significantly correlated with GPI-deficient granulocyte clone size only in the current analysis. Although fatigue was the most commonly reported symptom, its presence was not associated with GPIdeficient granulocyte clone size in either analysis, suggesting that fatigue may not be a relevant indicator of disease severity based on clone size.

Finally, QoL assessment results from FACIT-Fatigue scores and EORTC Global Health/QoL (median [Q1, Q3], $34.0[27.0,40.0]$ and $58.3[41.7,75.0]$, respectively) indicated patients in our analysis experienced considerable diseaserelated fatigue and impairment in overall QoL relative to normative reference scores reported for the general adult population (mean [SD], 43.6 (9.4) and 75.7 (21.2), respectively) [19, 32]. Future analyses to better understand the relationships between these patient-reported outcomes and disease burden would provide further insight into the impact of $\mathrm{PNH}$ on patients' health-related QoL.

Interpretation of our results may be mitigated by several limitations. The current analysis focused on patients with PNH who had not been treated with a complement inhibitor at the time of enrollment in the Registry, and we did not assess 
the potential impact of treatment on disease-related parameters. Because this analysis was based on an observational and noninterventional data set, not all patients had available data for every outcome assessed, and the current analysis did not account for changes in disease status that may have occurred prior to patients' enrollment in the Registry. Thus, patients' missing clone size data were not included in this analysis. Additional analyses are needed to investigate GPI-deficient granulocyte clone size and changes in clone size as potential prognostic markers for risk of clinical outcomes (e.g., MAVEs, including TEs). Given the heterogeneity in GPIdeficient granulocyte clone sizes observed in the current study, more robust prospective data are needed to confirm the best management approach for each individual patient with PNH. Also, comparisons across racial and/or ethnic subgroups were not conducted in the current study. The majority of patients in our analysis were white (78\%) and from Europe (68\%), with relatively small proportions of Asians (16\%) and blacks (3\%) represented in the study population. In a previous report of PNH Registry data from patients with PNH in South Korea, median granulocyte clone size was higher $(48.8 \%$ versus $31.8 \%$ ), as was median LDH ratio (4.1 versus 1.9 ) than the overall patient population in the current study [15]. Therefore, our observed results should not be extrapolated to race- or ethnicity-based subgroups.

In summary, consistent with previous analyses of patients in the PNH Registry, higher disease burden was generally associated with larger GPI-deficient granulocyte clone size in our study. However, substantial disease burden was still found in patients with GPI-deficient granulocyte clone size $<10 \%$. The presence of hemolysis in patients of all GPIdeficient granulocyte clone sizes is of particular clinical relevance because hemolysis is associated with significantly higher risk of TEs, the leading cause of mortality in PNH $[15,17,27]$. In this study, a noteworthy proportion of patients with GPI-deficient granulocyte clone size $<10 \%$ experienced HDA, MAVEs, RBC transfusions, impaired renal function, hemolysis, and $\mathrm{PNH}$-related symptoms, which might also be associated with the underlying BMF.

\section{Conclusions}

This International PNH Registry analysis is the largest PNH study to date to document the clinical characteristics, burden of disease, and potential trends in manifestations of PNH related to GPI-deficient granulocyte clone size. In general, patients with larger GPI-deficient granulocyte clone sizes had higher disease burden; however, many patients with smaller clone sizes had experienced clinically important medical events and associated symptoms. This study confirms the findings of a previous Registry analysis [6] and adds to our knowledge regarding the prevalence of HDA in Registry patients as well as the relationship between HDA and GPIdeficient granulocyte clone size in patients with PNH. The improved characterization of this rare, life-threatening disease and of relationships among clinical measures of interest in patients with PNH may better inform patient management.

Acknowledgments This analysis was sponsored by Alexion Pharmaceuticals, Inc., Boston, MA, USA. Alexion provided a scientific accuracy review of their data. Changes resulting from comments received were made by the authors on the basis of scientific and editorial merit. The sponsor had no influence on the interpretation of the data or reporting of the results. The decision to submit the manuscript was made independently by the authors with no influence from the sponsor. The sponsor and investigators thank the patients and their families for their participation in and support for this clinical study. The authors would like to acknowledge Peloton Advantage, LLC, an OPEN Health company, which provided editorial and medical writing support by Michael D. Morren, RPh, MBA, with funding from Alexion Pharmaceuticals, Inc., and Kirsten $\mathrm{Zu}, \mathrm{ScD}, \mathrm{PhD}$, of Alexion Pharmaceuticals, Inc., for additional statistical analysis support.

Data availability statement Qualified academic investigators may request participant-level, de-identified clinical data and supporting documents (statistical analysis plan and protocol) pertaining to this study. Further details regarding data availability, instructions for requesting information and our data disclosure policy will be available on the Alexion. com website (https://alexion.com/research-development).

Authors' contributions HS and JPM were responsible for the study concept and design, performed research and recruited patients, analyzed the data, assumed responsibility and accountability for data integrity and accuracy, and wrote the paper. AR, DJA, YK, LL, JMS performed research and recruited patients, analyzed the data, and wrote the paper. AW was responsible for the study concept and design, assumed responsibility and accountability for data integrity and accuracy, analyzed the data, was responsible for the statistical analysis, and wrote the paper. GS was responsible for the study concept and design, analyzed the data, and wrote the paper. All authors reviewed and approved the manuscript.

Funding information Open Access funding provided by Projekt DEAL. This study was funded by Alexion Pharmaceuticals, Inc.

\section{Compliance with ethical standards}

Conflict of interest Hubert Schrezenmeier has received honoraria and research support (both to University of Ulm) from Alexion Pharmaceuticals, Inc., and honoraria (to University of Ulm) from Ra Pharma, Roche, and Alnylam Pharmaceuticals. Alexander Röth has received honoraria and consulting fees from Alexion Pharmaceuticals, Inc., Roche, and Novartis, and research support from Alexion Pharmaceuticals, Inc., and Roche. David J. Araten has received honoraria and consulting fees from Alexion Pharmaceuticals, Inc. Yuzuru Kanakura has received honoraria and research support from Alexion Pharmaceuticals, Inc. Loree Larratt has received honoraria and research support (for the laboratory at the University of Alberta, for ADAMTS13 testing) from Alexion Pharmaceuticals, Inc. Jamile M. Shammo has received honoraria, consulting fees, and research support from Alexion Pharmaceuticals, Inc. Amanda Wilson and Gilda Shayan are former employees and stockholders of Alexion Pharmaceuticals, Inc. Jaroslaw P. Maciejewski has received consulting fees from Alexion Pharmaceuticals, Inc., Apellis Pharmaceuticals, and Ra Pharma; has also received speaker 
fees from Alexion Pharmaceuticals, Inc. and is a member of the Executive Committee of International PNH Registry for Alexion Pharmaceuticals, Inc.

Research involving human participants and/or animals The Registry protocol was approved by an ethics committee for each site, and the Registry is being conducted in compliance with the International Council for Harmonisation Guideline for Good Clinical Practice.

Informed consent The institutional review boards (or equivalent) of participating centers approved the Registry, and written informed consent was provided by all patients before study entry.

Open Access This article is licensed under a Creative Commons Attribution 4.0 International License, which permits use, sharing, adaptation, distribution and reproduction in any medium or format, as long as you give appropriate credit to the original author(s) and the source, provide a link to the Creative Commons licence, and indicate if changes were made. The images or other third party material in this article are included in the article's Creative Commons licence, unless indicated otherwise in a credit line to the material. If material is not included in the article's Creative Commons licence and your intended use is not permitted by statutory regulation or exceeds the permitted use, you will need to obtain permission directly from the copyright holder. To view a copy of this licence, visit http://creativecommons.org/licenses/by/4.0/.

\section{References}

1. Hillmen P, Lewis SM, Bessler M, Luzzatto L, Dacie JV (1995) Natural history of paroxysmal nocturnal hemoglobinuria. N Engl J Med 333(19):1253-1258. https://doi.org/10.1056/ nejm199511093331904

2. Brodsky RA (2014) Paroxysmal nocturnal hemoglobinuria. Blood 124(18):2804-2811. https://doi.org/10.1182/blood-2014-02522128

3. Jang JH, Kim JS, Yoon SS, Lee JH, Kim YK, Jo DY, Chung J, Sohn SK, Lee JW (2016) Predictive factors of mortality in population of patients with paroxysmal nocturnal hemoglobinuria $(\mathrm{PNH})$ : results from a Korean PNH registry. J Korean Med Sci 31(2):214-221. https://doi.org/10.3346/jkms.2016.31.2.214

4. Parker C, Omine M, Richards S, Nishimura J, Bessler M, Ware R, Hillmen P, Luzzatto L, Young N, Kinoshita T, Rosse W, Socie G (2005) Diagnosis and management of paroxysmal nocturnal hemoglobinuria. Blood 106(12):3699-3709. https://doi.org/10.1182/ blood-2005-04-1717

5. Parker CJ (2011) Management of paroxysmal nocturnal hemoglobinuria in the era of complement inhibitory therapy. Hematology Am Soc Hematol Educ Program 2011:21-29 https://doi.org/10. 1182/asheducation-2011.1.21

6. Schrezenmeier H, Muus P, Socie G, Szer J, Urbano-Ispizua A, Maciejewski JP, Brodsky RA, Bessler M, Kanakura Y, Rosse W, Khursigara G, Bedrosian C, Hillmen P (2014) Baseline characteristics and disease burden in patients in the International Paroxysmal Nocturnal Hemoglobinuria Registry. Haematologica 99(5):922929. https://doi.org/10.3324/haematol.2013.093161

7. Socie G, Mary JY, de Gramont A, Rio B, Leporrier M, Rose C, Heudier P, Rochant H, Cahn JY, Gluckman E (1996) Paroxysmal nocturnal haemoglobinuria: long-term follow-up and prognostic factors. French Society of Haematology. Lancet 348(9027):573577

8. Nishimura J, Kanakura Y, Ware RE, Shichishima T, Nakakuma H, Ninomiya H, Decastro CM, Hall S, Kanamaru A, Sullivan KM,
Mizoguchi H, Omine M, Kinoshita T, Rosse WF (2004) Clinical course and flow cytometric analysis of paroxysmal nocturnal hemoglobinuria in the United States and Japan. Medicine (Baltimore) 83(3):193-207

9. Young NS, Meyers G, Schrezenmeier H, Hillmen P, Hill A (2009) The management of paroxysmal nocturnal hemoglobinuria: recent advances in diagnosis and treatment and new hope for patients. Semin Hematol 46(1 Suppl 1):S1-S16. https://doi.org/10.1053/j. seminhematol.2008.11.004

10. Hill A, Kelly RJ, Hillmen P (2013) Thrombosis in paroxysmal nocturnal hemoglobinuria. Blood 121(25):4985-4996. https://doi. org/10.1182/blood-2012-09-311381

11. Moyo VM, Mukhina GL, Garrett ES, Brodsky RA (2004) Natural history of paroxysmal nocturnal haemoglobinuria using modern diagnostic assays. Br J Haematol 126(1):133-138. https://doi.org/ 10.1111/j.1365-2141.2004.04992.x

12. Loschi M, Porcher R, Barraco F, Terriou L, Mohty M, de Guibert S, Mahe B, Lemal R, Dumas PY, Etienne G, Jardin F, Royer B, Bordessoule D, Rohrlich PS, Fornecker LM, Salanoubat C, Maury S, Cahn JY, Vincent L, Sene T, Rigaudeau S, Nguyen S, Lepretre AC, Mary JY, Corront B, Socie G, Peffault de Latour R (2016) Impact of eculizumab treatment on paroxysmal nocturnal hemoglobinuria: a treatment versus no-treatment study. Am J Hematol 91(4):366-370. https://doi.org/10.1002/ajh.24278

13. Risitano AM, Rotoli B (2008) Paroxysmal nocturnal hemoglobinuria: pathophysiology, natural history and treatment options in the era of biological agents. Biologics 2(2):205-222

14. Hall C, Richards S, Hillmen P (2003) Primary prophylaxis with warfarin prevents thrombosis in paroxysmal nocturnal hemoglobinuria (PNH). Blood 102(10):3587-3591. https://doi.org/10.1182/ blood-2003-01-0009

15. Lee JW, Jang JH, Kim JS, Yoon SS, Lee JH, Kim YK, Jo DY, Chung J, Sohn SK (2013) Clinical signs and symptoms associated with increased risk for thrombosis in patients with paroxysmal nocturnal hemoglobinuria from a Korean Registry. Int J Hematol 97(6): 749-757. https://doi.org/10.1007/s12185-013-1346-4

16. Almeida AM, Bedrosian C, Cole A, Muus P, Schrezenmeier H, Szer J, Rosse WF (2017) Clinical benefit of eculizumab in patients with no transfusion history in the international paroxysmal nocturnal haemoglobinuria registry. Intern Med J 47(9):1026-1034. https://doi.org/10.1111/imj.13523

17. Yenerel MN, Muus P, Wilson A, Szer J (2017) Clinical course and disease burden in patients with paroxysmal nocturnal hemoglobinuria by hemolytic status. Blood Cells Mol Dis 65:29-34. https://doi. org/10.1016/j.bcmd.2017.03.013

18. Aaronson NK, Ahmedzai S, Bergman B, Bullinger M, Cull A, Duez NJ, Filiberti A, Flechtner H, Fleishman SB, de Haes JC et al (1993) The European Organization for Research and Treatment of Cancer QLQ-C30: a quality-of-life instrument for use in international clinical trials in oncology. J Natl Cancer Inst 85(5):365-376

19. Cella D, Lai JS, Chang CH, Peterman A, Slavin M (2002) Fatigue in cancer patients compared with fatigue in the general United States population. Cancer 94(2):528-538. https://doi.org/10.1002/ cncr. 10245

20. Yellen SB, Cella DF, Webster K, Blendowski C, Kaplan E (1997) Measuring fatigue and other anemia-related symptoms with the Functional Assessment of Cancer Therapy (FACT) measurement system. J Pain Symptom Manage 13(2):63-74

21. Hill A, Rother RP, Wang X, Morris SM Jr, Quinn-Senger K, Kelly R, Richards SJ, Bessler M, Bell L, Hillmen P, Gladwin MT (2010) Effect of eculizumab on haemolysis-associated nitric oxide depletion, dyspnoea, and measures of pulmonary hypertension in patients with paroxysmal nocturnal haemoglobinuria. Br J Haematol 149(3):414-425. https://doi.org/10.1111/j.1365-2141.2010.08096. $\mathrm{x}$ 
22. Pu JJ, Mukhina G, Wang H, Savage WJ, Brodsky RA (2011) Natural history of paroxysmal nocturnal hemoglobinuria clones in patients presenting as aplastic anemia. Eur J Haematol 87(1):37-45. https://doi.org/10.1111/j.1600-0609.2011.01615.x

23. Peffault de Latour R, Mary JY, Salanoubat C, Terriou L, Etienne G, Mohty M, Roth S, de Guibert S, Maury S, Cahn JY, Socie G (2008) Paroxysmal nocturnal hemoglobinuria: natural history of disease subcategories. Blood 112(8):3099-3106. https://doi.org/10.1182/ blood-2008-01-133918

24. Richards SJ, Norfolk DR, Swirsky DM, Hillmen P (1998) Lymphocyte subset analysis and glycosylphosphatidylinositol phenotype in patients with paroxysmal nocturnal hemoglobinuria. Blood 92(5):1799-1806

25. Borowitz MJ, Craig FE, Digiuseppe JA, Illingworth AJ, Rosse W, Sutherland DR, Wittwer CT, Richards SJ (2010) Guidelines for the diagnosis and monitoring of paroxysmal nocturnal hemoglobinuria and related disorders by flow cytometry. Cytometry B Clin Cytom 78(4):211-230. https://doi.org/10.1002/cyto.b.20525

26. Sharma VR (2013) Paroxysmal nocturnal hemoglobinuria: pathogenesis, testing, and diagnosis. Clin Adv Hematol Oncol 11(Suppl $13(9)): 2-8$

27. Rother RP, Bell L, Hillmen P, Gladwin MT (2005) The clinical sequelae of intravascular hemolysis and extracellular plasma hemoglobin: a novel mechanism of human disease. JAMA 293(13): 1653-1662. https://doi.org/10.1001/jama.293.13.1653

28. Parker CJ (2016) Update on the diagnosis and management of paroxysmal nocturnal hemoglobinuria. Hematology Am Soc Hematol Educ Program 2016(1):208-216. https://doi.org/10.1182/ asheducation-2016.1.208
29. Scheinberg P, Marte M, Nunez O, Young NS (2010) Paroxysmal nocturnal hemoglobinuria clones in severe aplastic anemia patients treated with horse anti-thymocyte globulin plus cyclosporine. Haematologica 95(7):1075-1080. https://doi.org/10.3324/ haematol.2009.017889

30. Hillmen P, Elebute M, Kelly R, Urbano-Ispizua A, Hill A, Rother RP, Khursigara G, Fu CL, Omine M, Browne P, Rosse W (2010) Long-term effect of the complement inhibitor eculizumab on kidney function in patients with paroxysmal nocturnal hemoglobinuria. Am J Hematol 85(8):553-559. https://doi.org/10.1002/ajh. 21757

31. Villegas A, Nunez R, Gaya A, Cuevas-Ruiz MV, Bosch JM, Carral A, Arrizabalaga B, Gomez-Roncero MI, Mora A, Bravo P, Lavilla E, Monteserin C, Hernandez B, Martinez-Barranco P, Jarque I, Urquia MA, Garcia-Donas G, Brunet S, Gonzalez FA, Urbano A (2017) Presence of acute and chronic renal failure in patients with paroxysmal nocturnal hemoglobinuria: results of a retrospective analysis from the Spanish PNH Registry. Ann Hematol 96(10): 1727-1733. https://doi.org/10.1007/s00277-017-3059-x

32. Hinz A, Singer S, Brahler E (2014) European reference values for the quality of life questionnaire EORTC QLQ-C30: results of a German investigation and a summarizing analysis of six European general population normative studies. Acta Oncol 53(7):958-965. https://doi.org/10.3109/0284186x.2013.879998

Publisher's note Springer Nature remains neutral with regard to jurisdictional claims in published maps and institutional affiliations. 